\title{
Revisiting the Technology Challenges and Proposing Enhancements in Ambient Assisted Living for the Elderly
}

\author{
Karoly Bozan \\ Duquesne University \\ bozank@duq.edu
}

\author{
Andrew Berger \\ Care Alliance Health Center \\ aberger@carealliance.org
}

\begin{abstract}
Several social and technical trends support the elderly's desire to live independently in their preferred environment, despite their increasing medical needs, and enhance their quality of life at home. Ambientassisted living (AAL) has the capabilities to support the elderly and to decrease their dependency on formal or informal caregivers. We provide a review of the technological challenges that were identified as inhibiting factors in the past decade and then present recent technological advances, e.g., cloud computing, machine learning, artificial intelligence, the Internet of Things. We also fill the gap in the current literature in regard to specific AAL solutions and propose fourthgeneration AAL technology design. We find that most informal caregivers are family members who are medically untrained and that the use of advanced analytical processes on AAL-generated data could significantly increase symptom identification. We also present the implications and remaining challenges along with recommendations for future research.
\end{abstract}

\section{Introduction}

The increase in the number of aging adults, 65 years and older, is a common demographic characteristic of most industrialized countries. In the United States, there are more than 46 million elderly adults, around 1 in 7 people, and this figure is projected to double in the next three decades [1]. Over $80 \%$ of these older adults choose to stay in their own homes and to maintain their independence and quality of life by choice or because they cannot afford other options [2]. An AARP telephone survey of 2,250 adults aged 60 years and older found that $90 \%$ of respondents want to "age in place" and that $85 \%$ do not plan to make modifications to their homes to do so [3].

A survey conducted by the Kungsholmen Project, which involved 1,099 elderly individuals, found that a significant portion of the elderly population suffers from common diseases, such as hypertension (38\%), dementia (21\%), and heart failure (18\%) [4]. These and other diseases are more risky and costly to treat, with decreased effectiveness, if medical attention is delayed [5]. Therefore, independent living among the elderly can pose a risk to their health. These risks can be mitigated by a nearby caretaker or family member, but the majority of elderly do not have such an individual available. Those who do are receiving care from the estimated over 43 million immediate family members [6] who provide care for their aging relatives and another 15 million caregivers who care for relatives with Alzheimer's or dementia [7]. Notably, prevention and proactive care as well as in-home recovery for the elderly are not only less costly but also are preferred and increase patient satisfaction [8].

Demographic change, coupled with the need to accommodate the elderly's preference to "age in place," has led to significant and interrelated changes and advances in healthcare technologies. Academia and practice have an interest in the development of products (hardware and software), interrelated systems, and multi-stakeholder solutions to support this increasing demand from elderly populations as well as their caretakers and healthcare providers. As a range of evolving products with diverse features and functionalities have been made available in the marketplace, it has become more challenging to understand their purpose or the needs that they serve and the utilization of their technology [9]. Recent technology innovations call for a revisiting of these products and services as related to the needs of the users.

The scarce availability of human assistance has led to home environments that may be equipped with sensors and their systems that can determine whether a person has fallen or is not performing his or her daily routine [10-11]. This technology can provide continuous and unobtrusive monitoring and not only benefits those being monitored but also their loved ones, such as immediate family members, who are the informal caregivers [12]. This intelligent and unobtrusive, yet ever-present, support to individuals 
embedded in their environment is known as ambientassisted living (AAL). This interdisciplinary (health, gerontology, social sciences, and information technology) term is widely used in academic research and in the industry, even though there is no widely accepted definition for it [13]. Muñoz et al. [14] cites the traits of AAL as the use of information communication technology or interconnected assisting devices to enable individuals to remain active and socially connected while living independently as elderly individuals. The more advanced technical characteristics include sensors, smart interfaces, and artificial intelligence [15].

The early years of AAL saw limited technological capabilities, heavily reliance on patient input, and provision of only basic support, such as appointment or medication reminders. In recent years, advancements in technology have resulted in an interconnected network that can monitor and provide support for the elderly and aid both formal and informal caretakers [16].

The most recent wave of technological innovations has not only disrupted the services and products that software development organizations create but also has had an impact on the processes used to develop these products. We evaluate the opportunities that technical innovations create for AAL technology. We also review several major caretaker forums and evaluate unmet needs through text mining of public posts. In this way, we develop an understanding of technical capability and then propose design considerations related to how the needs of caregivers, especially medically untrained family members, can be met to better care for their aging loved ones.

This study is structured as follows. We first present the literature on the early years and related technical challenges of AAL. We then review recent technical advancements and their impact on AAL and related concepts, such as ambient intelligence and smart home. Next, using text mining and analysis, we focus on the caretaker forums to evaluate the main issues that caretakers face. We then propose design considerations and technical solutions related to these challenges, especially those that involve safety and aiding the recognition of the symptoms of potentially serious medical conditions, using the latest technology within AAL. Finally, we discuss the implications for software development firms, healthcare providers, and other stakeholders, followed by a presentation of future technical challenges and recommendations for related research.

\section{The evolution of ambient assisted living technology and its challenges 2.1. Evolution of AAL technology}

AAL is rooted in (1) traditional assisted-living technologies designed for people with disabilities; (2) design approaches for accessibility, usability, and acceptability of interactive technology; and (3) ambient intelligence, which provides intelligent and unobtrusive, yet ever-present, support to individuals. AAL technology currently has the ability to anticipate and respond to the changing needs of the elderly and adapt to the heterogeneous nature of the needs of the elderly and their caretakers. This complex support is the result of an evolved ecology of the above assistive technologies.

Through their systematic literature review, Blackman et al. [17] identified three generations of AAL technologies. Table 1 provides a summary of their findings:

\begin{tabular}{|c|c|c|}
\hline First-gen. AAL & Second-gen. AAL & Third-gen. AAL \\
\hline ALARM & DETECT/REPORT & PREVENT \\
Wearable device: & Home sensors: & Integration of \\
Response to & Automatic & home sensors \\
emergency & response to & and wearable \\
Requires user to & emergencies and & devices: \\
initiate alarm & detection of & Prevention, \\
& hazards & monitoring, and \\
& May feel intrusive & assistance \\
& & Less obtrusive \\
\hline
\end{tabular}

Table 1. Three generations of AAL based on Blackman et al. (2015) [17]

First-generation devices consisted mainly of response systems, usually a wearable, button-triggered alarm. Such devices required conscious enabling from the user. An obvious disadvantage is seen in the case in which the user is unable to trigger the alarm.

Second-generation AAL technology addressed the limitations of the devices of the previous generation. Devices in this era detected emergency situations by sensors that could determine an instance of falling or an environmental hazard, such as a gas leak or excessive heat or cold. These sensors also provide comfort for those with difficulties with using household appliances. The weakness of these devices was their intrusiveness.

Devices in the third generation of AAL incorporate the most advanced technology and, as a result, are capable of preventing problems in addition to detecting and reporting. These devices can be considered a form of ambient intelligence, as they are unobtrusive and monitor lives in a sensible and responsive manner [18]. 


\subsection{Challenges of AAL technology}

Several challenges have been identified for the early generations of AAL in terms of technical capabilities [19]:

- Dynamic service availability: the uneven availability of informal caregivers and their supporting infrastructure. To mitigate the problem of dynamicity, Sun et al. [19] recommended the use of service-oriented architecture due to its flexible, standardized architecture and its ability to enable the connection of various services.

- Service mapping: challenge with mapping the available/requested services, specifically, the lengthy time to map and the burdensome memory requirements.

- Real-time unconformity check: the need for pattern learning and anomalous behavior.

- Technological frustration: the need for userfriendly interfaces or seamless wearable technology.

\section{Technical innovations and their impact on ambient assisted living}

\subsection{Recent technical innovations}

Information technology (IT) innovations are enabled by exponential improvements in computing speed, data storage, processing capability, transfer rate, and display of information. The technological components, such as software or hardware, that are new to the industry or to adopters, are often augmented by other innovations within organizations, including new forms of cognition, meaning, work processes, organizational structure, and business processes. These interrelated innovations establish new development capabilities and formulate new products and services, the way they are used, and the environment in which they are applied [20].

The following fundamental changes in the base technical capability from the past decade are [21]:

- The increased rate of data transfer in distributed computing, which is an enabler of access to innovative software development platforms that generate large volumes of data transfer and sharing among devices.

- Cloud computing, which Accenture defines as the dynamic provisioning of IT capabilities (hardware, software, or services) from third parties over a network. The three layers of the cloud computing stack are: (1) Infrastructure as a Service (IaaS), the "building blocks" of cloud IT, such as scalable grids or clusters of virtualized servers to support the data center needs of an organization; (2) Platform as a Service (PaaS), which provides virtualized servers for users to run applications or develop new ones without maintaining the underlying infrastructure; and (3) Software as a Service (SaaS), a cloud model that delivers on-demand applications, usually on a subscription basis, with applications as hosted and managed by the cloud provider.

- Mobile computing, which enables the access and modification of data through a mobile device over a mobile network.

These fundamental changes enable architectural changes and novel software development methods and the development of novel generic features, such as:

- Microservices architecture. This has recently emerged as a leading software application development frame that assumes the presence of a suite of independently deployable, small, modular services that are "glued" together for an application. Each service runs through a unique language-independent process and communicates through a well-defined, lightweight interaction protocol, e.g., HTTP/REST, JSN, protobuf.

- Integrated programming environment.

- Real-time decision support based on a large amount of data generated by GPS and sensors with the use of artificial intelligence and machine learning.

- Event-driven APIs. As compared to the early 2000s, APIs now have a different and more prominent role. Earlier, they were seldom used across organizational boundaries when organizations adopted Java or Apache with JavaBean plugins on their preset libraries. Currently, any service can become connected to smart objects through API, e.g., the use of Alexa to respond to complex questions through APIs from multiple source of data. API was previously a critical element of architecting frameworks such as J2EE. Currently, it is a quick way of creating and connecting building blocks that emerge as new kinds of software ecologies.

- The Internet of Things, which connects smart devices and "things" into networked systems.

- Augmented reality, the interactive experience of real-world and computer-generated elements, using a collection of sensors. 


\subsection{Technical challenges of AAL and the impact of technical innovations}

The identified technical innovations and their architectural impact can enhance AAL technological capabilities and respond to unmet needs or previous technical limitations, as seen below:

- Dynamic service availability: The need for dynamicity can be met with the microservice architectural style, particularly because more specialized microservices, which can be added to the existing application, are available. Bellmont et al. [22] used rapid deployment methods in AAL environments that utilized agile framework and microservices.

- Service mapping: Mapping needs can be met through remote procedural calls (RPC), JSON-RPC, and XML-RPC formats used for application programming interface (API) calls to fulfill the API delivery strategy requirements. Representative State Transfer (REST) provides a simple and fast method of accessing web services with no extensive messages or memory requirements. In AAL software, API calls are increasingly important when coupled with microservices and when relying on cloud computing.

- Real-time unconformity check: The combination of cloud computing and API calls, real-time validation of sensor-generated data, and predefined triggers can be implemented in AAL systems. An automated behavior analysis system identifies anomalous behavior that detects and acts upon changing health status based on standard behavioral patterns [23]. Accelerometer-generated data in conjunction with vital signs also can be used to detect anomalous behavior and trigger a response to adverse situations [24]. Fall detection systems are based on real-time analysis of data that deviates from normal behavior and can be widely used through wearable devices in conjunction with cloud computing and API calls [25,26]. These anomaly-detection approaches use the combination of cloud computing, machine learning, and various algorithms.

- Technology frustration: With the increased usability of user interfaces and the proven effectiveness of training of the [27], this challenge can be overcome but takes some effort from the users. Non-obtrusive approaches, such as sensors and wearable devices, do not require user interaction and should be used in situations in which users have limited capabilities to interact with the devices.

\section{Unmet needs and the technical capabilities}

The technological advancements and incremental enhancements of AAL devices and services have created a complex and supportive AAL ecosystem to enhance the quality of life of the elderly. Nevertheless, some needs still go unmet. In our early attempts to collect information through interviews, we realized that caretakers and their loved ones do not know their AAL needs, as they have limited knowledge of the technical capabilities, especially the latest innovations.

Our next approach to find unmet needs was to review posts of caretaker forums and evaluate the type of questions that caretakers post on these community knowledge-sharing and peer-support platforms [28]. Our pilot study found that, predominantly, immediate family members post and that their questions are related to safety and being able to receive immediate notification on anomalous behavior, e.g., falling, staying in bed too long, or changes in vital signs. Further, their difficulty with recognizing serious symptoms (e.g. stroke, heart attack) was evident in their questions.

To further analyze caretaker forum posts, we scraped three major forums and analyzed the posts through Linguistic Inquiry and Word Count (LIWC) analyses to answer the following research questions:

What are the main themes of major caretaker forums, and what are the relationships of those posting about their cared ones?

What type of AAL technology may be able to address the most pressing needs?

\subsection{Method}

We obtained data from the main caregiver forums that Google search returned and that were used frequently by a large group of its members. We developed a Python-based web crawler and ran a pilot study by scrapping 500 public posts. We randomized the posts retrieved with their posted solutions. All identifiers were removed, and only the time of the posts was kept, which ranged from August 2016 to January 2018. The returned posts were reviewed by two "interpreters" who captured and categorized the need indicated (directly or indirectly) in the posts and the responses given to the need. The posted need and 
the responses then were sorted into major categories based on the findings of the pilot-study.

The web crawler was developed based on Scrapy, a popular web-scraping tool written in Python (https://scrapy.org) a free, extensible, open-source framework for web scraping. We tested the categories created by a few random post scrapings, and those that did not fit into the initially established categories were collected in a "misc" category for further analysis.

Another 2,000 random posts were collected and further analyzed by two reviewers. The emotional analysis indicates posters' emotions and, possibly, frustration in regard to the situation for which they need solutions. We used the LIWC system $[29,30]$ to determine an emotional score, which can range from 0 to 100 , indicating exceptionally negative to exceptionally positive, with a no-particular-emotion range of 40-60. Outside of the 40-60 tone rating range indicates an emotional post, with negative below 40 and positive above 60 .

Interrater reliability was checked using Cohen's Kappa to measure the degree to which the two raters concur in their sorting; -1 indicates complete disagreement, 0 indicates agreement by chance, and 1 indicates perfect agreement. The confidence interval (CI) also was calculated for the obtained Cohen's Kappa, as kappa is an estimate of interrater reliability, and CIs are of interest based on standard error (SE). We subtracted the SE of the kappa of the desired standard error level, which is usually the $95 \% \mathrm{CI}$. Thus, we calculated the $\mathrm{CI}$ at $95 \%$, as follows:

$\mathrm{CI}_{\mathrm{L}}: \mathrm{k}+1.96 \times \mathrm{SE}_{\mathrm{k}}$

$\mathrm{CI}_{\mathrm{H}}: \mathrm{k}+1.96 \times \mathrm{SE}_{\mathrm{k}}$

where $\mathrm{k}=$ Cohen's Kappa, $\mathrm{SE}_{\mathrm{K}}=$ standard error of kappa.

\subsection{Results}

We initially analyzed all of the posts and responses and then focused on the post content that could be helped by technical solutions embedded into AAL. We determined the top five problem categories and responses among the initial 500 posts and used them for the categorization of the 2,000 posts; these are summarized in Tables 2 and 3.

\begin{tabular}{|l|c|}
\hline Problem & Count \\
\hline Frustration, negative emotions & 293 \\
\hline Symptoms, medical-advice seeking, medications & 82 \\
\hline Legal, family matters & 41 \\
\hline Nursing homes & 15 \\
\hline Ethics & 6 \\
\hline
\end{tabular}

Table 2. Top five problem-related post categories

\begin{tabular}{|l|c|}
\hline Solution & Count \\
\hline $\begin{array}{l}\text { Information and recommended personal solutions } \\
\text { to posted problems }\end{array}$ & 174 \\
\hline Physician or professional advice & 61 \\
\hline Well-being of the caretaker or the elderly & 49 \\
\hline Assisted care facilities & 23 \\
\hline Safety & 18 \\
\hline
\end{tabular}

Table 3. Top five solution-related post categories

The emotional rating of the posts had a tone rating of 33.67, indicating moderately negative emotions, which is explained by the high number of "frustrated" posts by overwhelmed family members. Some even apologized and indicated that writing about the situation that upsets them already makes them feel better and that the supporting responses were very helpful. The responses demonstrated slightly positive emotions, indicated by the 62.48 tone rating.

The interrater reliability score indicated substantial agreement across problem posts rates, with a Cohen's Kappa of $0.619(p<0.01), 95 \%$ CI $(0.614,0.623)$, and moderate agreement for the solution post rates, with a kappa score of $0.493(p<0.001), 95 \%$ CI $(0.488$, $0.498)$.

\subsection{Post and response examples}

The research question in the forum content analysis concerned the main themes and the relationship between the elderly patients and those who took care of them.

We found that the vast majority of the posts (92\%) were in regard to immediate family members, such as parents or grandparents. This explains why they are posting on the forums, as professional caretakers are trained to handle most issues (e.g., frustration, emotional exhaustion) or are not involved in the described situation (e.g., inheritance, guilt, indecisiveness about home care vs. nursing home).

The leading topics in inquiries posted were seeking advice in handling general problems, emotional support, and opportunities to release frustration (Table 2 ). The most surprising symptom-related posts included somewhat general questions about seemingly innocent incidents, with a lack of awareness of potentially serious consequences. For example, a user posted about a situation in which her mother got up late at night to use the bathroom and fell. She described the episode in length, including "but as she was talking, her lips were ... weird. She even asked if she was talking funny. She said she felt like she was." Almost immediately she received responses about this detail, indicating that those are symptoms of a stroke and that she should take her mother to the hospital for an immediate checkup. 
Another post described how a caregiver's father with dementia had a weakness in his arm and became clumsy while feeling faint and out of breath: "He was getting angry not being able to turn the page in his paper due to that numbness in his arm and was fuming out breath." Again, responses called out the possibility of a heart attack, which the poster did not assume.

In another example, the caregiver described symptoms of serious conditions and sought advice on the forum instead of from healthcare professionals. This is further evidence that untrained family members ask basic health questions on the forum and are in need of technical support that checks for incidents and symptoms that may indicate serious health problems and that require immediate physician attention.

Several posts described the challenges of updating physicians on episodes of health problems and the challenge of documenting them. For example, a family member indicated, "I wanted to let my father's doctor know about the high blood pressure and his dizziness, but by the time I had a chance to talk to [his doctor], none of these symptoms were present and I don't know what else was I supposed to check."

The available devices that elderly patients can use appear in a number of posts, but family members felt that some devices could be more user friendly and possibly connected to the Internet: "Finally, my mother can measure her blood sugar, but I would like to see the reading remotely, as sometime she forgets to write the readings down by the time we speak."

Finally, several posts describe the concern over the safety of the loved one and of feeling helpless. Responses were very supportive and shared best practices and sound, practical advice. The available technical devices and AAL technology were mentioned in regard to social connections (e.g., friends, family, community), and respondents recommended mainly Facebook and Skype.

\section{Discussion}

We review the challenges and unmet needs of the elderly who live at home based on extracted posts and propose technical solutions to the identified needs. We also propose fourth-generation AAL technology with design considerations.

\subsection{Technical solution capabilities in the AAL setting}

The purpose of our public caretaker forum post analysis was to identify unmet needs by the use of enhanced technology embedded into an AAL ecosystem. The analysis found that caretakers are not familiar with signals of triggered episodes or potentially serious illnesses. Further, several posts indicated the need for remote monitoring of vital or other physical measures and possibly connecting to the electronic medical records of the elderly loved ones.

The previously identified recent base technology inventions and their combinational effect may offer solutions to these unmet technical needs. For example, machine learning and artificial intelligence, combined with the power of cloud computing, can not only learn the user's behavior and alert when there are anomalies but also can check for symptoms of stroke by analyzing facial movements. This solution could help a caretaker who does not know relevant symptoms. The technology could be a tablet application that monitors the facial gestures of users. The tablet has the highest acceptance rate by the elderly but depends on previous PC usage [31] and training received [27], especially from family [32]. Virtual assistants and voice activations also provide a way for the elderly to increase technology acceptance [33,34].

The concern about sharing patients' longitudinal health status with their primary physician can be addressed by interconnected technology. The National Institute of Standards and Technology defines interconnectivity as the direct connection of two or more systems for the purpose of data and information resources sharing. Providers often must make decisions without access to complete, up-to-date patient health history, to which a connected electronic health record (EHR) system be a potential remedy [35]. With proper security considerations, the AAL-embedded device or sensor-generated data could be made available to the patient's health record as patient-generated information. Ubiquitous sensing can be embedded into a variety of infrastructures and daily objects in an elderly person's house without requiring any user input. Contextual information about behavior can be collected and analyzed, using cloud computing. The concept of smart home includes the collection and response to such information without the need for direct input from the users. Wearable and embedded sensors capture time-series information about the user's vital signs, environment, movement, and body posture, and ambulatory activity can be captured through gyroscopes and accelerometers [36]. Sensordata processing is a complex task due to inevitable noise and missing data. Raw data needs to be processed and converted to a proper format for context identification, activity modeling, and preparation for unsupervised machine learning [36]. Figure 1 displays the sensor-data processing elements required for preparation for analysis and the results that are sent to the physician. Cloud computing and machine learning 
can provide the proper computing power and specialized applications for data processing and analysis.

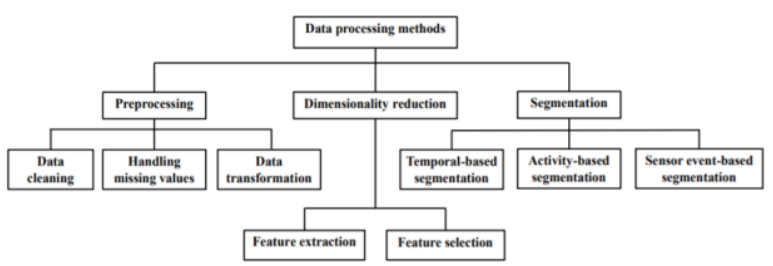

Figure 1: Sensor data-processing methods based on Ni et al. (2015) [36].

Similarly, the need for remote monitoring of different health information can be accomplished by the network of devices, following the Internet of Things-notion embedded into AAL. The collected and contextualized information can be sent to caretakers, even if they are remotely located, with real-time insight into the major indicators of safety and well-being of their loved one or person in their care. The enhanced user interface and mobile computing, coupled with artificial intelligence, can provide timely and relevant data for caretakers to aid with decision making and proper responses to symptoms that may have gone unnoticed.

The combination of (1) cloud computing, (2) machine learning, (3) Internet of Things, and (4) artificial intelligence can provide possible solutions to the caretakers' problems identified in recent forum posts. Thus, based on recent technology innovations, we extend [17] findings and propose the fourth generation of AAL technology that not only includes the latest base technological innovations but also responds to recognized user needs in a way that is easy to understand and based on a series of interconnected device-generated data. The caretaker-need analysis extends the literature, which most recently highlighted the need for evaluating new AAL solutions and developing better understanding of user needs [37]. Our fourth-generation AAL technology solutions represent the capabilities of Analyze / Connect / Communicate as a response to caretakers' needs.

This new generation of AAL technology includes real-time data analytics from sensor and devicegenerated data, which produce actionable insight to caretakers and other stakeholders while generating new knowledge on causes and effects using the big data concept. The connectivity includes the interconnected devices, following the Internet of Things concept, and the connectedness to the patient's EHR. Georges Matar et al. [28] proposes a sensor-embedded mattress that senses pressure and monitors the user's posture. The data can be analyzed, compared to behavioral trends, and identify anomalous behavior. These data may not indicate warning symptoms, but, coupled with data generated by other devices, sensors may determine correlations that are not obvious to elderly persons or their caretakers.

It is especially helpful to healthcare providers to be able to see multiple behavioral traits and their longitudinal correlations rather than hearing about only a single and obvious problem. For example, frequent urination at night is an obvious symptom, but having sudden swollen legs and increased blood pressure along with difficulty standing up from a sitting position (information found in an EHR) may warrant a different diagnosis than just the complaints about difficulty sleeping due to urinary frequency. The interconnected devices could highlight possible correlations, giving the physician more comprehensive and granular information on which to evaluate symptoms. More advanced analysis, including artificial intelligence and comparison of large data samples, could help with decision making, using statistical analysis that takes into account patient characteristics and previous cases, similar to the initiative of Explorys, a healthcare big data analytics firm.

The communication component of this new generation of AAL technology can create a channel to provide feedback to the elderly and their caretakers in a clear and understandable manner. Finally, an enhanced and user-friendly user interface could display the recommended actions.

\subsection{Implications}

AAL is based on ambient intelligence, working in an unobtrusive, adaptable, dynamic, interconnected, embedded, and intelligent environment [39]. This complex ecosystem of software and hardware creates a context-aware solution [40] and recognizes end-user needs [37]. Our study contributed to the knowledge of software development organizations by highlighting caretakers' needs and proposing design considerations of the systems and features that they develop.

The ultimate goal of AAL technology is to support the elderly to live safely and independently in their preferred home environment in the least intrusive manner and with user-friendly interfaces. The proposed combination of technology can enhance AAL features to more closely meet these goals. Advanced analytics based on AAL-generated big data and even predictive analytics can not only provide more granular information to healthcare providers but also can warn real-time whether a combination of symptoms warrants a physician's immediate attention. Elderly patients who stay in their own home depend on their caretakers' judgment as to which symptoms are serious enough to 
seek medical attention. Finally, the proposed use of the latest technology may significantly reduce the serious consequences that result from delayed medical help due to medically untrained caretakers.

\section{Challenges, limitations, and further research}

Several challenges need to be addressed to properly implement the proposed fourth-generation of AAL technology. First, the security of the collected data needs to be addressed [41], especially during the transmission to external databases for analytics and storage. Further, the legal aspects of the use of data [41] need to be carefully evaluated before large amounts of data generated by embedded and wearable sensors and other medical devices are collected and transferred for analytics.

Physicians need to be closely involved in the development of algorithms and analytical models that analyze large datasets and look for possible symptoms of serious illnesses. False alarms would lessen the credibility of an intelligent system; therefore, it is recommended to incrementally add more complex algorithms as data are collected and for machine learning to be trained and tested thoroughly. The new microservice-based software architecture allows for updates without users' involvement; their embedded devices would stay the same, and only the analytical stack would be regularly updated through releases over the network.

User participation is an obvious challenge, and there is a rich literature on individual adoption of technology among the elderly. Because the proposed AAL technology is an ecosystem of devices, sensors, and large data that are sent for analytics outside of the user's house, it may become more difficult to gain initial consent. Describing and demonstrating to the elderly the easily understood and actionable insights generated may be helpful but require significant effort, and further empirical validation is required for this type of technology acceptance.

Sharing the information with physicians requires a significant effort to develop possible standards or interfaces to automatically upload to designated sections of EHRs. Further, physician "buy-in" may be a challenge, as patient-generated data pose a risk to physicians, as they cannot check the validity of the information. Involving physicians in the design of data collection and analysis and ensuring that users are not interfering with the data but, rather, that they are automatically collected and cleaned may enhance physician trust. This is also an area that requires a sound theoretical approach and empirical validation from the physician's point of view.

Because the needs were identified in caretaker forums, visited primarily by family members, these needs may not be valid for other stakeholders of the care of the elderly. Professional caretakers and physicians may have diverse needs, and although the collected data may help them to a certain extent, their needs may call for data from additional sources. Further research is needed to better understand the data needs of other stakeholders. Most importantly, validating the needs of the elderly should be a concern. This may be challenging, however, as caretakers and elderly persons may have diverse expectations of AAL technology.

\section{Conclusion}

We reviewed the literature for previously unmet technical needs related to AAL. We found that recent technical innovations have the capacity to meet these needs. We also analyzed unmet needs by extracting 2,000 recent posts and their responses from public caretaker forums. The results showed that caretakers who post on these forums are predominantly untrained, immediate family members who are unfamiliar with the latest available technology that could support their needs when taking care of their loved ones.

We also analyzed the emotional aspect of the posts and responses and found that the emotional scores of the posts were mainly negative, whereas the responses were lightly positive, based on LIWD.

We proposed a technical solution to some of the problem areas that caretakers discussed in the forums. We also proposed a fourth-generation AAL technology that extends Blackman et al.'s [17] findings. Advancement in the available technology provides great support for the next generation of AAL technology, and this study highlighted the initial design considerations to develop this technology based on caretakers' needs.

\section{References}

[1]https://www.deseretnews.com/article/865675803/M ost-elderly-want-to-stay-in-their-homes-but-is-thatwhats-best.html Retrieved: January 30, 2018

[2] Van Hoof, J., Kort, H.S.M., Rutten, P.G.S., Duijnstee, M.S.H. "Ageing-in-place with the use of ambient intelligence technology: perspectives of older users” Int. J. Med. Inform., 80(5), 2011, pp. 310-331. [3] Phone $\quad$ Survey 2012
https://www.aarp.org/content/dam/aarp/livable- 
communities/learn/research/the-united-states-of-agingsurvey-2012-aarp.pdf

[4] Marengoni, A., Bengt, W., Karp, A., Fratiglioni, L. "Prevalence of Chronic Diseases and Multimorbidity Among the Elderly Population in Sweden", American Journal of Public Health, 98(7), 2008, pp. 1198-1200.

[5] BOFFETTA, P., et al. "The Consortium on Health and Ageing: Network of Cohorts in Europe and the United States (CHANCES) project - design, population and data harmonization of a large-scale, international study", European Journal of Epidemiology, 29(12), 2014, pp. 929-936.

[6] National Alliance for Caregiving and AARP. Caregiving in the U.S, National Alliance for Caregiving, Washington D.C . 2009.

[7] Alzheimer's Association. "Alzheimer's Disease Facts and Figures", Alzheimer's and Dementia, 10( 2), 2014.

[8] Ellenbecker, C.H., Samia, L., Cushman, M.J., et al. "Patient Safety and Quality in Home Health Care", In: Hughes RG, editor. Patient Safety and Quality: An Evidence-Based Handbook for Nurses. Rockville (MD): Agency for Healthcare Research and Quality (US); 2008, Chapter 13.

[9] Calvaresi, D., Cesarini, D., Sernani, P., Marinoni, M., et al. "Exploring the ambient assisted living domain: a systematic review", Journal of Ambient Intelligence and Humanized Computing, 8(2), 2017, pp. 239-257.

[10] Costa, A et al. "Advances and trends for the development of ambient-assisted living platforms.", Expert Systems, 34(2), 2017.

[11] Ni , Q., Hernando, A.B.G., de la Cruz, I.P. "The Elderly's Independent Living in Smart Homes: A Characterization of Activities and Sensing Infrastructure Survey to Facilitate Services Development", Sensors, 15, 2015(5), pp. 11312-11362. [12] Hossain, M.A., Ahmed, D.T. "Virtual caregiver: an ambient-aware elderly monitoring system", IEEE Trans. Inf. Technol. in Biomed, 16(6), 2014, pp. 1024 1031.

[13] Cardinaux, F., Bhowmik, D., Abhayaratne, C., Hawley, M. S. "Video based technology for ambient assisted living: a review of the literature", J. Ambient Intell. Smart Environ., 3(3), 2011, pp. 253 - 269.

[14] Muñoz, A., Augusto, J., Villa A., Botia, J. "Design and evaluation of an ambient assisted living system based on an argumentative multi-agent system", Pers. Ubiquitous Computing 15(4), 2011, pp. $377-387$.

[15] Sixsmith, A., Mueller, S., Lull, F., Klein, M. et al. "A user driven approach to developing ambient assisted living systems for older people: the SOPRANO project", in: Intelligent Technologies for
Bridging the Grey Digital Divide , J. Soar, R. Swindell and P. Tsang eds., IGI Global, Hershey PA, 2010.

[16] Lotfi, A., Langensiepen, C., Moreno, P.A., et al. "An Ambient Assisted Living Technology Platform for Informal Carers of the Elderly-iCarer", EAI Endorsed Transactions on Pervasive Health and Technology, 3(9), 2017.

[17] Blackman, S., Matlo, C., Bobrovitskiy, C. et al. "Ambient assisted living technologies for aging well: a scoping review", J. Intell. Syst., 25 (1), 2015, pp. 5569.

[18] Kleinberger, T., Becker, M., Ras, E., et al. "Ambient intelligence in assisted living: enable elderly people to handle future interfaces", in: Universal Access in HCI , C. Stephanidis ed., Part II, pp. 103 112, Springer-Verlag, Berlin, 2007.

[19] Sun, H., De Florio, V., Gui, N., Blondia. C. "Promises and Challenges of Ambient Assisted Living Systems", In Proceedings of the 2009 Sixth International Conference on Information Technology: New Generations (ITNG '09). IEEE Computer Society, Washington, DC, USA, 1201-1207. 2009.

[20] Lyytinen, K., Rose, G. M. "Disruptive Information System Innovation: The Case of Internet Computing", Information Systems Journal, 13(4), 2003, pp. 301-330.

[21] Bozan, K., Lyytinen, K., Rose, G., and Mount, G. 2018. "The Changing Landscape of Software Development: Building Schools of Goldfish, Not Thoroughbreds," in: Proceedings of the 17th AIS SIGSAND Symposium. Syracuse, NY.

[22] Bellmunt, J., Mokhtari, M., Abdulzarak, B., Aloulou, H. "Agile framework for rapid deployment in ambient assisted living environments", In Proceedings of the 18th International Conference on Information Integration and Web-based Applications and Services (iiWAS '16). 20016, ACM, New York, NY, USA, pp. 410-413.

[23] Ordóñez, F.J., de Toledo, P., Sanchis, A. "Sensorbased bayesian detection of anomalous living patterns in a home setting", Pers. Ubiquit. Comput., 19, 2014, pp. 259-270.

[24] Gjoreski, H., Rashkovska, A., Kozina, S., Lustrek, M., Gams, M. "Telehealth using ecg sensor and accelerometer", In: Proceedings of the 37 th International Convention on Information and Communication Technology, Electronics and Microelectronics (MIPRO), Opatija, Croatia, 26-30 May 2014; pp. 270-274.

[25] Rimminen, H., Lindstrom, J., Linnavuo, M., Sepponen, R. "Detection of falls among the elderly by a floor sensor using the electric near field", IEEE Trans. Inf. Technol. Biomed., 14(6), 2010, pp. 14751476. 
[26] Ghasemzadeh, H., Jafari, R., Prabhakaran, B. “A body sensor network with electromyogram and inertial sensors: Multimodal interpretation of muscular activities", IEEE Trans. Inf. Technol. Biomed., 14(2), 2010, pp. 198-206.

[27] Bozan, K., Mooney, D. "Intervention Study on the Effect of Training and Support on Perceived Level of Patient Portal Ease of Use Among the Elderly", In: Proceedings of the 51st Hawaii International Conference on System Sciences, Waikoloa, Hawaii, 2018, pp. 3292-3301.

[28] Scharett, E., Madathil, K.C., et al. "An Investigation of the Information Sought by Caregivers of Alzheimer's Patients on Online Peer Support Groups", Cyberpsychol Behav Soc Netw., 20(10), 2017, pp. 640-657.

[29] Kahn, J.H., Tobin, R.M., Massey, A.E., Anderson, J.A. "Measuring emotional expression with the 11 Linguistic Inquiry and Word Count", Am J Psychol., 120(2), 2007, pp. 263-286.

[30] Tausczik, Y.R., Pennebaker, J.W. "The Psychological Meaning of Words: LIWC and 13 Computerized Text Analysis Methods", J Lang Soc Psychol., 29(1), 2010, pp.24-54.

[31] Dahn, I., Ferdinand, P., Lachmann, P. "Supporting Senior Citizen Using Tablet Computers", In: Miesenberger, K., et al. (eds) Computers Helping People with Special Needs. ICCHP 2014. Lecture Notes in Computer Science, vol 8548. Springer, Cham pp. 320-330.

[32] Tsai, H.S, Shillair, R., Cotten, S.R. "Social Support and 'Playing Around':An Examination of How Older Adults Acquire Digital Literacy With Tablet Computers", Journal of Applied Gerontology, (36) 1, 2017, pp.29-55.

[33] Hammer, S., Kirchner, K., André, E., Lugrin, B. "Touch or Talk?: Comparing Social Robots and Tablet PCs for an Elderly Assistant Recommender System", In: Proceedings of the Companion of the 2017 ACM/IEEE International Conference on Human-Robot Interaction (HRI '17). 2017, ACM, New York, NY, USA, 129-130.

[34] Teixeira, A., Hamalainen, A., et al. "Speechcentric multimodal interaction for easy-to-access online services a personal life assistant for the elderly", In: Proc. DSAI 2013, pp. 389-397.

[35] Sands, D. Z., Wald, J.S. "Transforming Health Care Delivery Through Consumer Engagement, Health Data Transparency, and Patient-Generated Health Information." Yearbook of Medical Informatics 9.1, 2014, pp. 170-176.

[36] Ni, Q. N., García Hernando, A., and Ivan Pau de la Cruz, I. "The elderly's independent living in smart homes: A characterization of activities and sensing infrastructure survey to facilitate services development", Sensors, (15)5, 2015, pp.11312-11362. [37] Calvaresi, D., Claudi, A., Dragoni, A.F., Yu, E., Accattoli, D., Sernani, P. "A goal-oriented requirements engineering approach for the ambient assisted living domain", In: Proceedings of the 7th International Conference on Pervasive Technologies Related to Assistive Environments, Article 20, ACM, New York, NY, USA, PETRA '14, 2014, pp. 20:120:4.

[38] Matar, G., Lina, J., Kaddoum, G., Riley, A. "Internet of things in sleep monitoring: An application for posture recognition using supervised learning", in: Proc. IEEE HealthCom 2016, International Conference on E-health Networking, Application \& Services, 2016.

[39] Sadri, F. “Ambient intelligence: A survey”, ACM Comput. Surv., 43(4), 2011, pp. 36:1-36:66.

[40] Fenza, G., Furno, D., Loia, V. "Hybrid approach for context-aware service discovery in healthcare domain", J Comput Syst Sci, 78(4), 2012, pp. 12321247.

[41] Petersen, C., DeMuro, P. "Legal and Regulatory Considerations Associated with Use of PatientGenerated Health Data from Social Media and Mobile Health (mHealth) Devices.” Applied Clinical Informatics, 6(1), 2015, pp. 16-26. 